desnähe die Inftusion einer 10-procentigen Lösung das schon ganz erloschene Leben wieder zurückzurufen vermag:

6. Die Infusion einer dïnnen Lösung von $0,6-0,75$ pCt. setzt zwar in dem normalen Zustande des Thieres ebenso wie die von Wasser die Geschwindigkeit des Blutansflusses herab, aber ihre wiederbelebende Kraft bei Verblutung ist ausser Frage gestellt.

7. Ist vorher in die Gewebe des Körpers oder in die serösen Höhlen eine grössere Menge der dünnen Lösung eingeführt, so. bewirkt eine Blutentziehnng eine Steigerung der Geschwindigkeit.

8. Diese Beschleunigung fällt ebenso wie die durch die Infusion einer concentrirteren Lösung mit einer Verdünnung des Blutes zusammen, welche durch Aufnahme von Flüssigkeit in die Blutgefässe bewirkt wird; sie erfolgt aber langsamer und ist weniger beträchtlich.

\title{
Die mechanischen and die optischen Dichtigkeiten des Blutes, der Galle und der Milch, und der Wasserverdünnungen derselben.
}

Von

\section{G. Valentin.}

Diese Untersuchungen sollten zanächst die Frage beantworten, ob thierische Flüssigkeiten, die Schleim oder feste Körperchen als Gemengtheile enthalten, dieselben Beziehungen zwischen Eigenschwere und Brechungsverhältniss, wie man sie für eine Anzahl reiner Lösungen gefunden hat, darbieten oder nicht. Die an dem Blute gemachten Erfahrungen enthïllten noch eine auf den ersten Blick eigenthümliche Frscheinung, der eine weitere Bedeutung zükommen dürfte.

Ieh suchte mir so frische Flïssigkeiten, als möglich, für jede erste der Prüfungsreihen zu verschaffen. Das gesehlagene Blut war in zwei Fällen noch warm, als ich mich anschickte, seine Eigenschwere in unverdünntem Zustande zu bestimmen. Die Galle 
wurde ungefähr eine bis anderthalb Stunden nach dem Tode des Ochsen und die Milch eben so kurze Zeit nach dem Melken der Kuh zum ersten Male untersucht.

Das 25 Cubikcentimeter fassende specifische Gewichtsfläschchen, das zur Ermittelung der Eigenschwere diente, gab 25,004 gr als Mittelwerth des Gewichtes des destillirten Wassers, das es bei $15^{\circ} \mathrm{C}$. aufnahm. Ich machte immer drei Einzelbestimmungen einer jeden der untersuchten Fliissigkeiten, um eine wahrscheinliche Durchschnittszahl zu erhalten - eine Vorsicht, die mit Gemengtheilen vermischte Lösungen immer nöthig machen.

Zwei Geissler'sche Buretten mit Glashähnen, die sehr feine Ausflussröhren besassen, dienten zu den Volumensbestimmungen der Milch und des zu deren Verdïnnung genommenen destillirten Wassers. Da das geschlagene Blut und die schleimigte Galle den engen Ausgang des Hahnes zu verstopfen drohten, so nahm ich später für sie Fläschchen, die genau 25 Cabikcentimeter Rauminhalt bis zu einer Marke darboten. Die Mischung wurde jedes Mal durch fünf- bis zehnfaches Umkehren der Röhre, in der sie enthalten war, möglichst gleichartig gemacht. Ich wiederholte dieses vor jeder Einzelbestimmung der Eigenschwere und ehe ich die zur Ermittelung des Brechungsverhältnisses dienende Probe entnahm.

Die Untersuchungen des Blutes und der Galle wurden bei $20^{\circ}$ bis $25^{\circ} \mathrm{C}$. und die der Milch bei $20^{\circ}$ bis $22^{\circ} \mathrm{C}$. der Luft vorgenommen. Musste eine dieser Flüssigkeiten iiber Nacht des Vergleiches wegen stehen bleiben, so schiitzte ich sie möglichst vor Verdunstung durch Verkorken der enghalsigen Flasche oder der Röhre, in der sie sich befand.

Das Refractometer war so eingestellt, dass das zu den Verdiunnungen gebrauchte destillirte Wasser 1,33448 als Durchschnittswerth atis zehn Einzelbestimmungen des Brechungsverhältnisses gab. Ich fuhr in der Regel mit dem Wasserzusatze so lange fort, bis die letzte Flïssigkeit einen Ablenkungseoefficienten darbot, der um weniger als eine Einheit der dritten Decimale den des Wassers iibertraf. 
Die mechanischen und optischen Dichtigkeiten des Blutes, etc. $\quad \mathbf{5 6 1}$

\begin{tabular}{|c|c|c|c|c|}
\hline \multirow{3}{*}{$\begin{array}{l}\text { Versuchs } \\
\text { nummer }\end{array}$} & \multicolumn{4}{|c|}{ I. Noch warmes Kalbsblut. } \\
\hline & \multicolumn{2}{|c|}{ 'Dichtigkeit. } & \multicolumn{2}{|c|}{ Brechungsverhältniss. } \\
\hline & Einzelwerthe & Mittel & Grenzwerthe & Mittel \\
\hline \multirow[t]{4}{*}{1.} & Reines Blut & & & \\
\hline & 1,0518 & & & \\
\hline & 1,0518 & 1,05167 & 1,3512 & 1,34964 \\
\hline & 1,0514 & & 1,3485 & \\
\hline
\end{tabular}

2. 50 Volumenprocente Blut und 50 pCt. Wasser.

$\begin{array}{llll}1,0254 & & 1,3538 & \\ 1,0254 & 1,02500 & 1,352 \check{5} & 1,35328 \\ 1,0242 & & & \end{array}$

3. $25 \mathrm{pCt}$. Blat und $75 \mathrm{pCt}$. Wasser.

$\begin{array}{llll}1,0122 & & 1,3442 & \\ 1,0120 & 1,01207 & 1,3436 & 1,34396 \\ 1,0120 & & & \end{array}$

4. 12,5 pCt. Blut und 87,5 pCt. Wasser.

$\begin{array}{llll}1,0056 & & 1,3392 & \\ 1,0054 & 1,00547 & 1,3388 & 1,33904 \\ 1,0054 & & & \end{array}$

5. $6,25 \mathrm{pCt}$. Blut und $93,75 \mathrm{pCt}$. Wasser.

$\begin{array}{llll}1,00199 & & 1,3367 & \\ 1,00188 & 1,001917 & 1,3356 & 1,33610 \\ 1,00188 & & & \end{array}$

6. $3,125 \mathrm{pCt}$. Blut und $96,875 \mathrm{pCt}$. Wasser.

$\begin{array}{llll}1,00068 & & 1,3307 & \\ 1,00016 & 1,000307 & 1,3352 & 1,33467 \\ 1,00008 & & & \end{array}$

II. Dasselbe Blut 24 Stunden später.

Indessen in einer verkorkten Flasche mit langem Halse und bis in diesen gefüllt aufbewahrt. Nicht faulig riechend.

7. Reines Blut.

$\begin{array}{llll}1,0558 & & 1,3545 & \\ 1,0523 & 1,05140 & 1,3537 & 1,35404 \\ 1,0489 & & & \end{array}$

8. 50 Volumenprocente Blut und $50 \mathrm{pCt}$. Wasser.

$\begin{array}{cccc}1,0282 & & 1,3545 & \\ 1,0280 & 1,02700 & 1,3537 & 1,35376 \\ 1,0248 & & . & \end{array}$


VersuchsDichtigkeit.

Brechungsverhältniss.

nummer.

Einzelwerthe Mittel. Grenzwerthe. Mittel.

9. $\quad 25$ pCt. Blat and 75 pCt. Wasser.

$\begin{array}{llll}1,0118 & & 1,3453 & \\ 1,0118 & 1,01173 & 1,3442 & 1,34452 \\ 1,0116 & & & \end{array}$

10. 12,5 pCt. Blut and 87,5 pCt. Wasser.
1,0054
1,3380
1.0055
$1,00537 \quad 1,3371$
1,33742
$1,005 \%$

11. 6,25 pCt. B lut und 93,25 pCt. Wasser.

$\begin{array}{llll}1,00184 & & 1,3361 & \\ 1,00181 & 1,00178 & 1,3353 & 1,33556 \\ 1,00168 & & & \end{array}$

12. 3,125 pCt. Blut und 96,875 pCt. Wasser.

$\begin{array}{llll}1,00028 & & 1,3348 & \\ 1,00016 & 1,00019 & 1,3344 & 1,33462 \\ 1,00012 & & & \end{array}$

III. Noch warmes Schaafsblut.

13. Reines Blut.

$\begin{array}{llll}1,0412 & & 1,3496 & \\ 1,0410 & 1,04080 & 1,3484 & 1,34888 \\ 1,0402 & & & \end{array}$

14. 47 Volumenprocente Blut and 53 pCt. Wasser.

$\begin{array}{llll}1,0248 & & 1,3527 & \\ 1,0247 & 1,02467 & 1,3517 & 1,35219 \\ 1,0245 & & & \end{array}$

15. 22,4 p Ct. Blat and 77,6 pCt. Wasser.

$\begin{array}{llll}1,0112 & & 1,3434 & \\ 1,011 & 1,01043 . & 1,3415 & 1,34222 \\ 1,090 & & & \end{array}$

16. $11,2 \mathrm{pCt}$. Blut und $88,8 \mathrm{pCt}$. Wasser.

$\begin{array}{llll}1,0047 & & 1,3392 & \\ 1,0047 & 1,00467 & 1,3385 & 1,33888 \\ 1,0046 & & & \end{array}$

17. $5,6 \mathrm{pCt}$. B 1 ut und $94,4 \mathrm{pCt}$. Wasser.

$1,00292 \quad 1,3378$

$\begin{array}{llll}1,00284 & 1,00287 & 1,3369 & 1,33740\end{array}$

1,00284 
Die mechanischen und optischen Dichtigkeiten des Blutes, etc. 563

\begin{tabular}{|c|c|c|c|c|}
\hline \multirow{2}{*}{$\begin{array}{l}\text { Versuchs- } \\
\text { nummer. }\end{array}$} & \multicolumn{2}{|c|}{ Dichtigkeit. } & \multicolumn{2}{|c|}{ Brechungsverhältniss } \\
\hline & Einzelwerthe. & Mittel. & Grenzwerthe. & Mittel. \\
\hline 18. & $\begin{array}{c}2,8 \text { p Ct. B lut } \\
1,00126\end{array}$ & and 97 & $\begin{array}{c}, 2 \text { p Ct. Wass } \\
1,3365\end{array}$ & \\
\hline & $\begin{array}{l}1,00118 \\
1,00116\end{array}$ & 1,00120 & 1,3362 & 1,333630 \\
\hline 19. & $\begin{array}{c}1,4 \text { p Ct. B lu } \\
1,00032\end{array}$ & und 98 & $\begin{array}{c}, 6 \mathrm{pCt} . \text { Wasse } \\
1,3355\end{array}$ & \\
\hline & $\begin{array}{l}1,00032 \\
1,00010\end{array}$ & 1,000247 & 1,3351 & 1,33532 \\
\hline 20. & $\begin{array}{c}0,7 \text { p Ct. B lut } \\
1,00008\end{array}$ & und 99 & $\begin{array}{c}3 \text { p Ct. Wass } \\
1,3353\end{array}$ & \\
\hline & $\begin{array}{l}1,00008 \\
1,00008\end{array}$ & 1,00008 & 1,3344 & 1,33474 \\
\hline
\end{tabular}

Die Flïssigkeit Nr. 20 war noch stark roth gefärbt, löschte alle Farben des Spectrums eines Hoffmann'schen geraden Spectroskopes bis auf Roth in Dicken von 10 bis 25 Millimeter aus, gab die beiden Blutbänder in Schichten von $8 \mathrm{~mm}$ und verdunkelte dann den grössten Theil des übrigen Grün, das Blau und das Violett.

IV. Dasselbe Schafblut, 48 Stunden später.

Indessen in einer verkorkten, bis in den dïnnen Hals derselben reichenden Menge aufbewahrt. Faulig riechend.

Versuchs-
nummer. Einzelwerthe, $\overbrace{\text { Mittel. }}^{\text {Dichtigkeit. }}$ Grenzwerthe. $\underbrace{\text { Brechungsverhältniss. }}_{\text {Mittel. }}$

21. Reines Blat.

$\begin{array}{llll}1,0430 & & 1,3495 & \\ 1,0424 & 1,04223 & 1,3488 & 1,34912 \\ 1,0413 & & & \end{array}$

22. 50 Volumenprocente B1ut und 50 pCt. Wasser.

$\begin{array}{llll}1,0202 & & 1,3488 & \\ 1,0202 & 1,02010 & 1,3473 & 1,34794 \\ 1,0199 & & & \end{array}$

23. $25 \mathrm{pCt}$. Blut und $75 \mathrm{pCt}$. Wasser.

$\begin{array}{llll}1,0094 & & 1,8408 & \\ 1,0094 & 1,00937 & 1,3402 & 1,34050 \\ 1,0093 & & & \end{array}$


Versuchsnummer.

24. $12,5 \mathrm{pCt}$. B lut and $87,5 \mathrm{pCt}$. Wasser.

\begin{tabular}{llll}
1,0038 & \multicolumn{3}{c}{1,3373} \\
1,0038 & 1,00377 & 1,3365 & 1,33692 \\
1,0037 & & &
\end{tabular}

25. $6,25 \mathrm{pCt}$. Blut und 93,75 pCt. Wasser.

$\begin{array}{llll}1,0013 & & 1,3362 & \\ 1,0012 & 1,00123 & 1,3355 & 1,33584 \\ 1,0012 & & & \end{array}$

26. 3,125 pCt. Blut und 96,875 pCt. Wasser.

$\begin{array}{llll}1,0004 & & 1,3348 & \\ 1,0004 & 1,00033 & 1,3344 & 1,33462 \\ 1,0002 & & & \end{array}$

V. Blut eines vor einer halben bis einer ganzen Stunde getödteten Schweines.

27. Reines Blut.

$\begin{array}{llll}1,0660 & & 1,3538 & \\ 1,0657 & 1,06570 & 1,3526 & 1,35310 \\ 1,0654 & & & \end{array}$

28. 83,3 Volumenprocente Blut und 16,7 pCt. Wasser.

$\begin{array}{llll}1,0550 & & 1,3556 & \\ 1,0547 & 1,05413 & 1,3539 & 1,35470 \\ 1,0527 & & & \end{array}$

29. 71,4 pCt. B Iut und 28,6 pCt. Wasser.

\begin{tabular}{llll}
1,0468 & \multicolumn{3}{c}{1,3569} \\
1,0466 & 1,04660 & 1,3553 & 1,35588 \\
1,0464 & & &
\end{tabular}

30. 62,5 pCt. Blut und 37,5 pCt. Wasser.

$\begin{array}{llll}1,0412 & & 1,3606 & \\ 1,0406 & 1,04080 & 1,3592 & 1,36008 \\ 1,0406 & & & \end{array}$

31. 55,6 pCt. Blut und 44,4 pCt. Wasser.

$\begin{array}{llll}1,0364 & & 1,3578 & \\ 1,0363 & 1,03630 & 1,3572 & 1,35745 \\ 1,0362 & & & \end{array}$

32. $50 \mathrm{pCt}$. B1ut und $50 \mathrm{pCt}$. Wasser.

$\begin{array}{llll}1,0324 & & 1,3549 & \\ 1,0322 & 1,03160 & 1,3545 & 1,35476 \\ 1,0302 & & & \end{array}$


Die mechanischen und optischen Dichtigkeiten des Blutes, etc. $\quad 565$

Versuchs- Dichtigkeit. Brechungsverhältniss.

nummer. Einzelwerthe. Mittel. $\overbrace{\text { Grenzwerthe. Mittel. }}^{\text {. }}$

33. 45,5 pCt. Blut und 54,5 pCt. Wasser.

$\begin{array}{llll}1,0291 & & 1,3532 & \\ 1,0289 & 1,02890 & 1,3526 & 1,35300 \\ 1,0287 & & & \end{array}$

34. 33,3 pCt. BIut und 66,7 pCt. Wasser.

$\begin{array}{llll}1,0210 & & 1,3488 & \\ 1,0209 & 1,02093 & 1,3476 & 1,34813 \\ 1,0209 & & & \end{array}$

35. 16,7 pCt. Blut und 83,3 pCt. Wasser.

$\begin{array}{llll}1,0100 & & 1,3412 & \\ 1,0100 & 1,0100 & 1,3404 & 1,34066 \\ 1,0100 & & & \end{array}$

36. 8,4 pCt. Blut und $91,6 \mathrm{pCt}$. Wasser.

$\begin{array}{llll}1,0042 & & 1,3372 & \\ 1,0040 & 1,00393 & 1,3363 & 1,33688 \\ 1,0036 & & & \end{array}$

37. 4,2 pCt. Blut und 95,8 pCt. Wasser.

$\begin{array}{llll}1,0014 & & 1,3356 & \\ 1,0012 & 1,00123 & 1,3349 & 1,33530 \\ 1,0011 & & & \end{array}$

38. 2,1 pCt. Blut und 97,9 pCt. Wasser.

$\begin{array}{rrr}1,0001 ? & 1,0001 ? & 1,3347 \\ 1,3343 & 1,33418\end{array}$

VI. Dasselbe Schweinsblut, 24 Stunden später.

In einer mit engem Halse versehenen und bis zu diesem gefüllten and verkorkten Flasche indessen aufbewahrt. Sichtlich dunkler gefärbt, als in dem frischen Zustande. Fautlig riechend.

39. Reines Blut.

$\begin{array}{llll}1,0712 & & 1,3732 & \\ 1,0701 & 1,07043 & 1,3723 & 1,37278 \\ 1,0700 & & & \end{array}$

40. $62,5 \mathrm{pCt}$. Volumenprocente B1ut und $37,5 \mathrm{pCt}$. Wasser.

$\begin{array}{llll}1,0402 & & 1,3615 & \\ 1,0400 & 1,04033 & 1,3609 & 1,36120 \\ 1,0399 & & & \end{array}$

41. 50 pCt. Blut und 50 pCt. Wasser. $1,0322 \quad 1,3556$

$1,0322 \quad 1,03217 \quad 1,3548 \quad 1,35522$

1,0321 
VersuchsDichtigkeit.

Brechungsverhältniss.

nummer.

Einzelwerthe. Mittel. Grenzwerthe. Mittel.

42. 25 pCt. Blut und 75 pCt. Wasser.

$\begin{array}{llll}1,0152 & & 1,3448 & \\ 1,0066 & 1,0947 & 1,3445 & 1,34470 \\ 1,0066 & & & \end{array}$

43. 12,5 pCt. Blut und 87,5 pCt. Wasser.

$\begin{array}{llll}1,0072 & & 1,3398 & \\ 1,0067 & 1,00687 & 1,3395 & 1,33962 \\ 1,0067 & & & \end{array}$

44. $6,25 \mathrm{pCt}$. Blut und 93,75 pCt. Wasser.

1,0026

1,3366

$\begin{array}{llll}1,0024 & 1,00247 & 1,3362 & 1,33638\end{array}$

1,0024

45. 3,125 pCt. Blut und 96,875 pCt. Wasser.

$\begin{array}{llll}1,0004 & & 1,3348 & \\ 1,0003 & 1,00033 & 1,3344 & 1,33460 \\ 1,0003 & & & \end{array}$

B. Galle.

VII. Unfiltrirte Ochsengalle ein bis anderthalb Stunden nach dem Tode des Rindes. Dunkelbraun, Sehr schleimig.

46. Reine Galle.

$\begin{array}{llll}1,0304 & & 1,3548 & \\ 1,0300 & 1,02993 & 1,3544 & 1,35468 \\ 1,0294 & & & \end{array}$

47. 50 Volumenprocente Galle und 50 pCt. Wasser.

$\begin{array}{llll}1,0142 & & 1,3448 & \\ \mathbf{1}, 0141 & 1,01413 & 1,3442 & 1,34450 \\ 1,0141 & & & \end{array}$

48. $\quad 25 \mathrm{pCt}$. Galle und 75 pCt. Wasser.

$1,0069 \quad 1,3392$

$1,0068 \quad 1,00677 \quad 1,3384 \quad 1,33870$

1,0066

49. 12,5 pCt. Galle und 87,5 pCt. Wasser.

$1,0039 \quad 1,3365$

$1,0038 \quad 1,00367 \quad 1,3363 \quad 1,33636$

1,0033

50. $6,25 \mathrm{pCt}$. Galle und $93,75 \mathrm{pCt}$. Wasser.

$\begin{array}{cccc}1,0018 & & 1,3349 & \\ 1,0016 & 1,00160 & 1,3346 & 1,33476 \\ 1,0014 & & & \end{array}$


Die mechanischen und optischen Dichtigkeiten des Blutes, etc. $\quad \mathbf{5 6 7}$

VIII. Dieselbe Galle filtrirt, aber dessenungeachtet schleimigt. Noch am Tage des Todes des Thieres untersucht.

Versuchs-
nummer. $\overbrace{\text { Einzelwerthe. Mittel. }}^{\text {Dichtigkeit. }} \overbrace{\text { Grenzwerthe. }}^{\text {Brechungsverhältniss. }}$

51. Reine Galle.

$\begin{array}{llll}1,0436 & & 1,3546 & \\ 1,0434 & 1,04334 & 1,3544 & 1,35434 \\ 1,0433 & & & \end{array}$

52. 50 Volumenprocente Galle und 50 pCt. Wasser.

$\begin{array}{llll}1,0184 & & 1,3455 & \\ 1,0147 & 1,01583 & 1,3446 & 1,34516 \\ 1,0144 & & & \end{array}$

53. 25 pCt. Galle und 75 pCt. Wasser.

$\begin{array}{llll}1,0070 & & 1,3395 & \\ 1,0067 & 1,00670 & 1,3393 & 1,33942 \\ 1,0064 & & & \end{array}$

54. 12,5 pCt. Galle und 87,5 pCt. Wasser.

$\begin{array}{llcl}1,0031 & & 1,3366 & \\ 1,0030 & 1,00300 & 1,3364 & 1,33656 \\ 1,0029 & & & \end{array}$

55. 6,25 pCt. Galle und $93,75 \mathrm{pCt}$. Wasser.

$\begin{array}{llll}1,0011 & & 1,3354 & \\ 1,0010 & 1,00100 & 1,3352 & 1,33536 \\ 1,0009 & & & \end{array}$

IX. Noch warme Rindsgalle. Grün, wässeriger und weniger schleimig; als die vorige.

56. Reine Galle.

$\begin{array}{llll}1,0220 & & 1,3493 & \\ 1,0215 & 1,02167 & 1,3488 & 1,34908 \\ 1,0215 & & & \end{array}$

57. 50 Volumenprocente Galle und 50 pCt. Wasser.

$\begin{array}{llll}1,0107 & & 1,3416 & \\ 1,0106 & 1,01053 & 1,3413 & 1,34144 \\ 1,0103 & & & \end{array}$

58. $25 \mathrm{pCt}$. Galle und 75 pCt. Wasser.

$\begin{array}{llll}1,0001 & & 1,3385 & \\ 1,0054 & 1,00560 & 1,3778 & 1,33804 \\ 1,0053 & & & \end{array}$

E. Pfiüger, Archiv f* Physiologie. Bd, XXII. 
Versuchs-

Dichtigkeit.

Brechungsverhältniss.

nummer. Einzelwerthe. Mittel. Grenzwerthe. Mittel.

59. 12,5 pCt. Galle und 87,5 pCt. Wasser.

$\begin{array}{llll}1,0024 & & 1,3367 & \\ 1,0024 & 1,00237 & 1,3363 & 1,33648 \\ 1,0023 & & & \end{array}$

60. 6,25 pCt. Galle and 93,75 pCt. Wasser.

$\begin{array}{llll}1,0008 & & 1,3355 & \\ 1,0007 & 1,0007 & 1,3346 & 1,33510 \\ 1,0006 & & & \end{array}$

\section{Kuhmileh,}

ein bis zwei Stunden nach dem Melken erhalten.

X. Erste Probe der Milch.

61. Reine Milch.

$\begin{array}{llll}1,0329 & & 1,3592 & \\ 1,0328 & 1,03270 & 1,3477 & 1,34843 \\ 1,0324 & & & \end{array}$

62. 49,6 Volumenprocente Milch and 50,4 pCt. Wasser.

$\begin{array}{llll}1,0158 & & 1,3413 & \\ 1,0158 & 1,01567 & 1,3401 & 1,34087 \\ 1,0154 & & & \end{array}$

63. 24,7 p Ct. Milch und 75,3 p Ct. Wasser.

$\begin{array}{llll}1,00784 & & 1,3383 & \\ 1,00780 & 1,00760 & 1,3364 & 1,33640 \\ 1,00752 & & & \end{array}$

64. 12,4 pCt. Milch und 87,6 p Ct. Wasser.

$\begin{array}{llll}1,0036 & & 1,3358 & \\ 1,0035 & 1,00353 & 1,3341 & 1,23471 \\ 1,0035 & & & \end{array}$

65. 6,2 pCt. Milch und 93,8 pCt. Wasser.

$\begin{array}{llll}1,0014 & & 1,3348 & \\ 1,0012 & 1,00127 & 1,3341 & 1,33445 \\ 1,0012 & & & \end{array}$

XI. Andere Milch.

66. Reine Milch.

$\begin{array}{llll}1,0314 & & 1,3491 & \\ 1,0312 & 1,003123 & 1,3473 & 1,34833 \\ 1,0811 & & & \end{array}$


Die mechanischen und optischen Dichtigkeiten des Blutes, etc.

Versuchs-

Dichtigkeit.

Brechungsverhältniss.

nummer.

Einzelwerthe. Mittel. $\overbrace{\text { Grenzwerthe. Mittel. }}$

67. 49,3 Volumenprocente Milch und 50,7 pCt. Wasser.

$\begin{array}{llll}1,0148 & & 1,3424 & \\ 1,0148 & 1,01473 & 1,3413 & 1,34186 \\ 1,0146 & & & \end{array}$

68. 24,5 pCt. Milch und 75,5 pCt. Wasser.

$\begin{array}{llll}1,0068 & & 1,3383 & \\ 1,0067 & 1,00660 & 1,3374 & 1,33784 \\ 1,0066 & & & \end{array}$

69. 12,2 pCt. Milch und 87,8 pCt. Wasser.

$\begin{array}{llrl}1,0028 & & 1,3358 & \\ 1,0028 & 1,00280 & 1,3353 & 1,335149 \\ 1,0028 & & & \end{array}$

70. 6,1 pCt. Mileh und 93,9 pCt. Wasser.

$\begin{array}{llll}1,0012 & & 1,3357 & \\ 1,0010 & 1,00103 & 1,3346 & 1,33500 \\ 1,0009 & & & \end{array}$

XIII. Andere Mileh.

71. Reine Miloh.

$\begin{array}{llll}1,0298 & & 1,3515 & \\ 1,0297 & 1,02973 & 1,3504 & 1,35086 \\ 1,0297 & & & \end{array}$

72. 59,3 Volumenprocente Milch und 40,7 pCt. Wasser.

$\begin{array}{llll}1,0144 & & 1,3424 & \\ 1,0144 & 1,01417 & 1,3418 & 1,342019 \\ 1,0137 & & & \end{array}$

73. 29,2 pCt. Milch and 70,8 pCt. Wasser.

$\begin{array}{llll}1,0067 & & 1,3394 & \\ 1,0066 & 1,00663 & 1,3384 & 1,33885 \\ 1,0066 & & & \end{array}$

74. 14,5 $\mathrm{pCt}$. Milch und 85,5 pCt. Wasser.

$\begin{array}{llll}1,0029 & & 1,3373 & \\ 1,0026 & 1,00257 & 1,3363 & 1,33676 \\ 1,0022 & & & \end{array}$

75. 7,2 pCt. Milch und 92,8 pCt. Wasser.

$\begin{array}{rrrr}1,0011 & & 1,3352 & \\ 1,0008 & 1,00090 & 1,3344 & 1,33476 \\ 1,0008 . & & & \end{array}$


Diese Untersuchungen lehren:

1. Die an dem Harne angestellten Beobachtungen 1) fithrten schon zu dem Schlusse, dass das Kochen und der hierdurch erzeugte Wasserverlust die Eigenschwere nachdrücklicher als das Brechungsverhältniss ändern. Dasselbe wiederholt sich für die Wasserdünnungen des Blntes, so dass auch die Eigenschwere desselben die Aenderungen kräftiger, als das Brechungsverhältniss anzeigt. Die Galle und die Milch bestätigten das Gleiche.

2. Die Eigenschwere und das Ablenkungsverhältniss sinken im Allgemeinen um so mehr, je grösser die Wasserprocente der Mischung werden. Beide wechseln in der Regel in gleichem Sinne. Der künstliche Zusatz einer wässerigen Eiweisslösung zu Menschenharn lieferte aber schon einen Fall in friberen Untersuchungen ${ }^{2}$ ), in welchem die Eigensehwere um 0,014 herunterging; während das Brechungsverhältniss um 0,0016 stieg. Das frische Blut zeigt diese entgegengesetzte Aenderung in verhältnissmässig bedeutenderem Grade, so lange nicht die Wasserverdünnung desselben eine gewisse Grenze ïberschritten hat.

Stellen wir nun zunächst die Zahlen, welche die ganz frischen, geschlagenen Blutmassen des Kalbes und des Schaafes gegeben haben, zusammen, so haben wir:

\begin{tabular}{|c|c|c|c|c|c|c|}
\hline Blutart. & 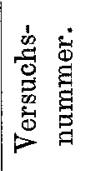 & $\begin{array}{l}\text { Zusammen- } \\
\text { setzung. }\end{array}$ & $\begin{array}{l}\text { Eigen- } \\
\text { schwere. }\end{array}$ & Unterschied. & 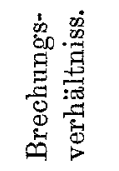 & Unterschied. \\
\hline Kalb & $\begin{array}{l}1 \\
2 \\
3\end{array}$ & $\begin{array}{l}\text { Reines Blut. } \\
50 \% \text { Blut und } \\
50 \% \text { Wasser. } \\
25 \% \text { Blut und } \\
75 \% \text { Wasser. }\end{array}$ & $\begin{array}{l}1,05167 \\
1,02500 \\
1,01207\end{array}$ & $\begin{array}{l}-0,02667 \\
-0,01293\end{array}$ & $\begin{array}{l}1,34964 \\
1,35328 \\
1,34396\end{array}$ & $\begin{array}{l}+\overline{0,00364} \\
-0,00932\end{array}$ \\
\hline Schaaf & $\begin{array}{l}13 \\
14 \\
15\end{array}$ & $\begin{array}{l}\text { Reines Blut. } \\
47 \% \text { Blut und } \\
58 \% \text { Wasser. } \\
22,4 \% \text { Blut und } \\
77,6 \% \text { Wasser. }\end{array}$ & $\mid \begin{array}{l}1,04080 \\
1,02467 \\
1,01043\end{array}$ & $\begin{array}{l}-\overline{0,01613} \\
-0,01424\end{array}$ & $\begin{array}{l}1,34888 \\
1,35219 \\
1,34222\end{array}$ & $\begin{array}{r}-\overline{0}, 00331 \\
-0,00997\end{array}$ \\
\hline
\end{tabular}

Während also die Eigenschwere durch die Verdünnung mit dem ungefähr gleichen Theile Wasser auf nahezu die Hälfte sank,

1) Dies Arch. Bd. XVII. S. 279.

2) a. a. O. S. 277. 279. 
stieg das Ablenkungsverhältniss um 1/448 der ursprünglichen Grösse. Es hatte aber wiederum einen kleineren Werth, wenn die Mischung $3 / 4$ ihres Rauminhaltes Wasser enthielt.

Da es sich unter diesen Verhältnissen fragte, bei welcher Verdünnung die grösste Erhöhung des Ablenkungsverhältnisses eintritt, so ging ich mit schwächeren Wasserzusätzen in dem Schweinsblute allmählig vorwärts. Es fand sich:

\begin{tabular}{|c|c|c|c|c|c|c|}
\hline Blutart. & 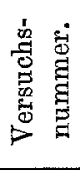 & $\begin{array}{l}\text { Zusammen- } \\
\text { setzung. }\end{array}$ & $\begin{array}{c}\text { Eigen- } \\
\text { schwere. }\end{array}$ & Unterschied. & 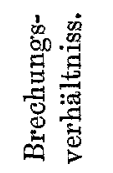 & Unterschied. \\
\hline \multirow{7}{*}{ Schwein } & 97 & eines Blut & 106570 & 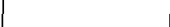 & 195010 & \\
\hline & 28 & $83,3 \%$ Blut und & 1,05413 & $-0 . \overline{01167}$ & 1,35470 & +000160 \\
\hline & & $17 \%$ Wasser. & & & & \\
\hline & 29 & $\begin{array}{r}71,4 \% \text { Blut und } \\
28,6^{\circ} \% \text { Wasser }\end{array}$ & 1,04660 & $-0,00753$ & 1,35588 & $+0,00118$ \\
\hline & 30 & $62,5 \%$ Blut und & 1,04080 & $-0,00580$ & 1,36008 & $+0,00420$ \\
\hline & 31 & $55,6 \%$ Blut und & 1,03630 & $-0,00450$ & 1,35745 & $-0,00263$ \\
\hline & 32 & $\begin{array}{l}44,4 \% \text { Wasser. } \\
50 \% \text { Blut und } \\
50 \% \text { Wasser. }\end{array}$ & 1,03160 & $-0,00470$ & 1,35476 & $-0,00269$ \\
\hline
\end{tabular}

Die grösste Zunahme des Brechungsverhältnisses, die 0,00698 oder $7 / 1353$, also ungefähr $1 / 193$ des ursprünglichen Werthes betrug, trat bei einer Wassermenge von $37,5 \%$ oder etwas mehr als $1 / 3$ des Gehaltes an Wasser ein. $44,4 \%$ oder beinahe $9 / 20$ führte schon zu einer merklichen Verkleinerung des Ablenkungsverhältnisses.

Vergleichen wir hiermit die Werthe, welche dieselben Blutarten 24 oder 48 Stunden später geliefert haben, so werden sie uns den Schlïssel zur wahrscheinlichen Erklärung jener Eigenthiumlichkeit des frischen Blates liefern. Wir haben:

\begin{tabular}{|c|c|c|c|c|c|c|c|}
\hline Blutart. & 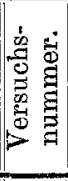 & 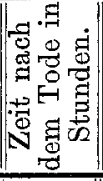 & $\begin{array}{c}\text { Zusammen- } \\
\text { setzung. }\end{array}$ & $\begin{array}{c}\text { Eigen- } \\
\text { schwere. }\end{array}$ & Unterschied. & 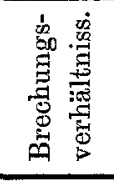 & Unterschied. \\
\hline $\begin{array}{r}\text { Kalb } \\
\text { Schaaf }\end{array}$ & $\begin{array}{r}7 \\
8 \\
21 \\
22\end{array}$ & $\begin{array}{l}24 \\
48\end{array}$ & 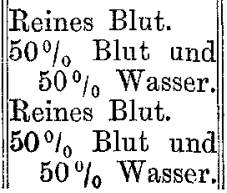 & $\begin{array}{r}1,05140 \\
1,02700 \\
1,04223 \\
1,02010\end{array}$ & $\begin{array}{l}-0,02440 \\
-\overline{0,02213}\end{array}$ & $\begin{array}{l}1,35404 \\
1,35376 \\
1,34912 \\
1,34794\end{array}$ & $\begin{array}{l}-\overline{0,00028} \\
-\overline{0,00148}\end{array}$ \\
\hline
\end{tabular}




\begin{tabular}{|c|c|c|c|c|c|c|c|}
\hline Blutart. & 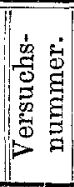 & 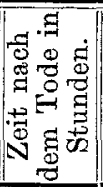 & $\begin{array}{c}\text { Zusammen- } \\
\text { setzung. }\end{array}$ & $\begin{array}{c}\text { Eigen } \\
\text { schwere. }\end{array}$ & Unterschied. & 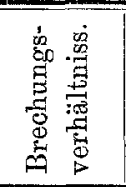 & Unterschied. \\
\hline Schwein & $\begin{array}{l}39 \\
40 \\
41\end{array}$ & 24 & $\mid \begin{array}{c}\text { Reines Blut. } \\
62,5 \% \text { Blut und } \\
37,5 \% \text { Wasser. } \\
50 \% \text { Blut und } \\
50 \% \text { Wasser. }\end{array}$ & $\begin{array}{l}1,07043 \\
1,04033 \\
1,03217\end{array}$ & $\begin{array}{l}-0,03010 \\
-0,00816\end{array}$ & $\begin{array}{l}1,37278 \\
1,36120 \\
1,35522\end{array}$ & $\begin{array}{l}-\overline{0,01158} \\
-0,00598\end{array}$ \\
\hline
\end{tabular}

Stellen wir die in dieser Tabelle verzeichneten Werthe denen der letzten und der vorletzten gegeniiber, so sehen wir, dass dieselben Verdünnangsgrade, welche das Brechungsverhältniss des frischen Blutes erhöhten, sie in dem 24 oder 48 Stunden alten Blute erniedrigen. Es ergibt sich ferner, dass das reine Blut ein grösseres Ablenkungsverhältniss als das noch frisch gebliebene nach ein bis zwei Tagen darbot. Wir haben in dieser Hinsicht:

\begin{tabular}{|c|c|c|c|c|}
\hline \multirow{3}{*}{ Blutart. } & \multicolumn{4}{|c|}{ Brechungsverhältniss. } \\
\hline & \multirow{2}{*}{$\begin{array}{c}\text { Frisches } \\
\text { Blut. }\end{array}$} & \multicolumn{2}{|c|}{ Blut } & \multirow{2}{*}{$\begin{array}{c}\text { Erhöhung } \\
\text { in dem } \\
\text { älteren } \\
\text { Blute. }\end{array}$} \\
\hline & & $\begin{array}{l}\text { nach } 24 \\
\text { Stunden. }\end{array}$ & $\begin{array}{l}\text { nach } 48 \\
\text { Stunden. }\end{array}$ & \\
\hline Kalb & 1,34964 & 1,35404 & \multirow{3}{*}{1,34912} & $+0,00440$ \\
\hline Schaaf & 1,34888 & & & $+0,00024$ \\
\hline Schwein & 1,35310 & 1,37278 & & $+0,01968$ \\
\hline
\end{tabular}

Ein Zusatz von $50 \%$ Wasser hatte das Brechungsverhältniss des ganz frischen Kalbsblutes um 0,00364 und den des Schaafsblutes um 0,00331 und ein solcher von 37,5\% Wasser den des Schweinsblutes um 0,00698 (letzteres als höchsten Werth) erhöht.

Alle diese Thatsachen erklären sich durch die Annahme, dass ein Anflösungsprocess in dem geschlagenen Blute in den ersten 24 Stunden and wahrscheinlich noch früher vor sich geht. Dieser Umstand vergrössert das Ablenkungsverhältniss. Die Eigenschwere wächst gleichzeitig mehr oder minder. Setzt man eine gewisse, bis zu einer bestimmten Grenze reichende Wassermenge dem ganz frischen Blate zu, so löst sich jener die Lichtbrechung verstärkende Körper auf. Das Brechungsverhältniss wächst, wenn auch die Eigenschwere bedeutend sinkt. 
Die Vermuthung liegt nahe, dass diejenige Verbindung, welche diese Veränderungen bewirkt, das Hämoglobin oder der Farbstoff der Blutkörperchen sei. Diese sind aber noch in grösster Menge mikroskopisch nachweisbar, wenn das Ablenkungsverhältniss den böchsten Werth in dem älteren Blute erreicht hat. Die Zahlen, die das Schaafblut lieferte, scheinen anzudeuten, dass später eine Zersetzung der Blutmasse eintreten kann, durch welche das Brechungsverhältniss wiederum von seiner grössten Höhe heruntergeht. Diese Aenderungen der geschlagenen Blutmasse nach dem Tode müssen bei feineren physikalischen und chemischen Untersuchungen derselben beruicksichtigt werden.

Frühere Beobachtungen ${ }^{1}$ ) ergaben schon, dass die Blutkörperchen einen nur sehr untergeordneten Einfluss auf das Brechungsverhältniss der Blutmasse ausuiben. Die eben erläuterten Thatsachen unterstützen dieses. Ist der die Brechung verstärkende Körper in den Blutkörperchen des frischen Blutes enthalten, so liesse sich nicht einsehen, weshalb es dann ein geringeres $\mathrm{Ab}$ lenkungsverhältniss besitzt, als die ältere Blatmasse und erst die Auflösung ihn wirksam machen soll, wenn die Blutkörperchen einen entscheidenden Einfluss auf die Grösse der Lichtbrechung besässen.

3. Nennen wir $V_{1}$ und $V_{2}$ die Volumina der beiden mit einander gemischten Flïssigkeiten und $\mathrm{V}_{3}$ den Rauminbalt der hierdurch erzengten Mischung, $S_{1}, S_{2}$ und $S_{3}{ }^{2}$ ) die entsprechenden Eigenschweren und setzen voraus, dass das Gewicht der Mischnng: der Summe ihrer beiden Bestandtheile gleicht, so haben wir:

$$
V_{3} S_{3}=V_{1} S_{1}+V_{2} S_{2}
$$

Also

$$
S_{3}=\frac{V_{1} S_{1}+V_{2} S_{2}}{V_{3}}
$$

Findet keine Dichtigkeitsänderung statt, so muss $V_{3}=V_{1}+V_{2}$ sein, also

$$
S_{3}=\frac{V_{1} S_{1}+V_{2} S_{2}}{V_{1}+V_{2}}
$$

1) Dies Arch. Bd. XIX. 1879. S. 98. 99.

2) Ich wähle hier und im Folgenden $S$ statt des von den Physikern and Chemikern oft gebrauchten $\partial$, um jede Verwechselung mit Differentialen zu vermeiden. 
Man hat hingegen eine Zu- oder Abnahme der Dichtigkeit, eine Condensation oder das Gegentheil derselben, einen positiven oder negativen Dichtigkeitswechsel, je nachdem:

$$
V_{3} \leq V_{1}+V_{2}
$$

Die eine Misehungsflüssigkeit war in unserem Falle destillirtes Wasser, das in einer Beindorf'schen Vorrichtung erhalten worden. 25 Cubikcentimeter derselben wogen, wie erwähnt, im Durchschnitt von zehn Einzelbestimmungen $25,004 \mathrm{gr}$ bei $+15^{\circ} \mathrm{C}$. Nimmt man den Rauminhalt des Wassers im Zustande seiner grössten Dichtigkeit oder bei $+4^{\circ} \mathrm{C}$. als Einheit, so beträgt er bei $+15^{0}$ C. nach Despritz 1,0008751. Die 25 Cubikcentimeter Wasser würden hiernach $25,0044 \mathrm{gr}$. bei $+4^{0} \mathrm{C}$. gewogen haben. Die Annabme, dass ein Cubikcentimeter desselben einem Gramm entspricht, liefert unter diesen Verhältnissen so kleine Unterschiede, dass sie noch längst innerhalb der Grenzen der möglichen Beobachtungsfehler liegen.

Setzen wir daher $S_{2}=1,00$, gibt (3)

$$
S_{3}=\frac{V_{1} S_{1}+V_{2}}{V_{1}+V_{2}}
$$

Ich befolgte in den meisten Verdünnungen des Blutes und der Galle den Grundsatz, dass ich gleiche Volumina der unmittelbar vorher verzeichneten Flïssigkeit und des Wassers mischte, dass also $V_{1}=V_{2}$ war. Fand keine Dichtigkeitsänderung statt, so verwandelte sich hierdurch (4) in

$$
S_{3}=1 / 2\left(S_{1}+1\right)
$$

wobei $S_{1}$ die Eigenschwere derjenigen Flüssigkeit bezeichnet, die nicht aus blossem destillirtem Wasser bestand. Stimmte die hiernach berechnete Dichtigkeit mit der gefundenen nicht überein, war diese vielmehr kleiner, so hatte man eine negative Dichtigkeitsänderung, eine Volumensvergrösserung in Folge der Mischung. Ist die Gleichung (5) anwendbar, so müssen die Decimalen der Eigenschwere einer folgenden Flüssigkeit oder Mischung der Hälfte der der vorhergehenden gleichen. Man wird finden, dass dieses in der Regel nicht zutraf, dass die berechnete Eigenschwere in den bei Weitem meisten Fällen grösser, als die gefundene ausfällt und die Ausnahmen, die besonders bei starken Verdünnungen vorkommen, wahrscheinlicher Weise auf Beobachtungsfehlern beruhen. Die Mischungen des Blutes (mit Ausnahme der unter Nro. 2 er- 
wähnten Erscheinungen, die natïrlich nicht hierhergezogen werden können), der Galle und der Milch mit Wasser liefern also als Regel einen negativen Dichtigkeitswechsel oder eine Zunahme des Rauminhaltes.

Um nicht zu weitläufig zu werden, verzeichne ich tabellariseh nur die Einzelwerthe je einer der mit jeder der drei Flüssigkeiten angestellten Versuchsreihen. Wir haben dann:

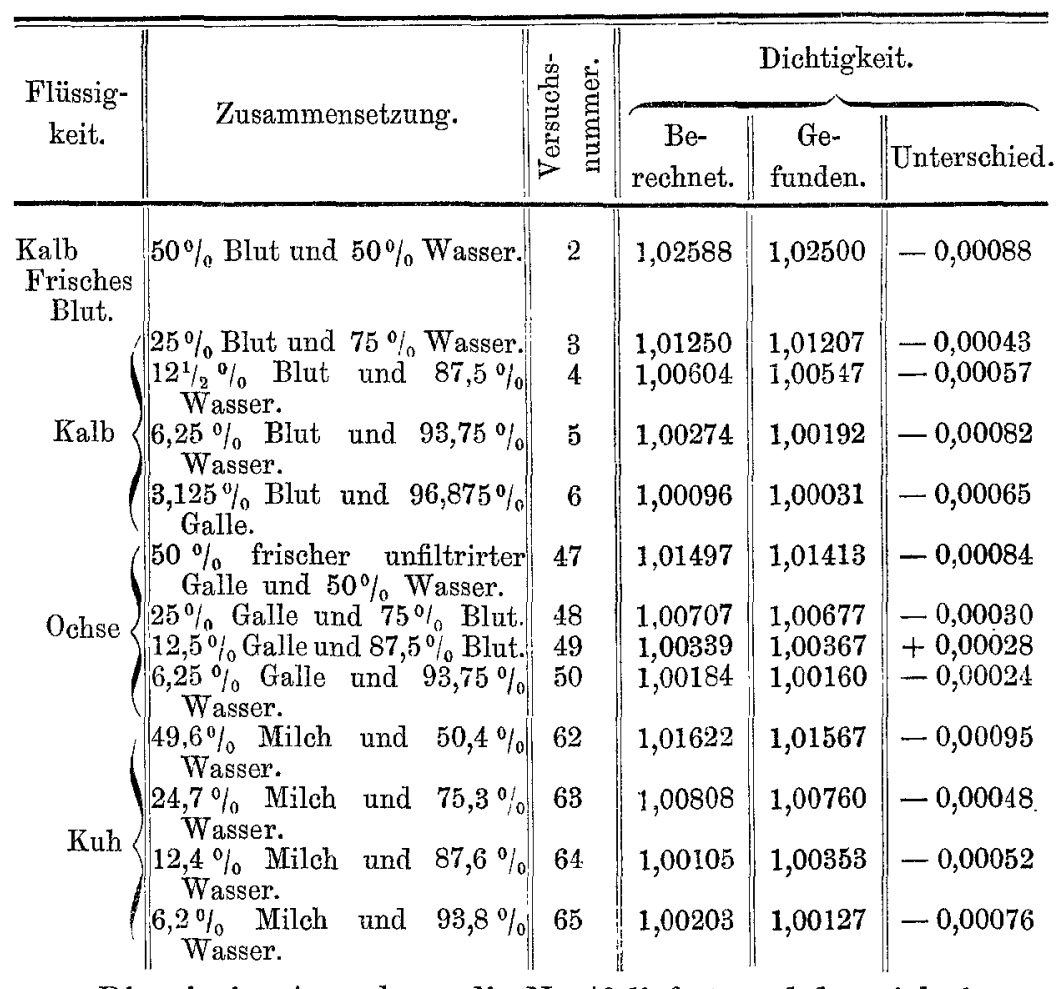

Die einzige Ausnahme, die Nr. 49 liefert, und der nicht immer regelmässige Gang der Abnahme mit dem Wachsthume der Wasserverdünnung diirfte von Beobachtungsfehlern zu einem nicht geringen Theile herriibren, da die Unterschiede in der vierten Decimale der Eigenschwere liegen.

Bedenkt man, dass wir es hier mit Mengungen von reinen Fliissigkeiten mit ungelösten Gebilden, wie dem Schleime, oder den Blutkörperchen, oder mit Körpern, die dem Einflusse des Wassers so gut als gänzlich widerstehen, wie den Milchkörperchen zu thun haben, so verdünnt die linzugesetzte Wassermenge wesentlich nur die vorhandene Lösung, also einen blossen Theil der 
Gesammtmasse. Sie tritt also in einer verhältnissmässig grösseren Menge linzu und drïckt deshalb die Eigensehwere herab.

4. Ich setzte die Wasserverdïnnung fort, bis das Brechungsverhältniss der Mischung dem des Wassers oder dem Werthe 1,33448 nahe kam. Stellen wir uns die hierher gehörenden Zahlen übersichtlich zusammen, so erhalten wir :

\begin{tabular}{|c|c|c|c|c|}
\hline Thier. & Zusammensetzung. & $\begin{array}{l}\text { Versuchs- } \\
\text { nummer. }\end{array}$ & $\begin{array}{l}\text { Brechungs- } \\
\text { verhältniss. }\end{array}$ & $\begin{array}{l}\text { Unterschied } \\
\text { von dem } \\
\text { Brechungsver- } \\
\text { hältniss des } \\
\text { Wassers. }\end{array}$ \\
\hline $\mathrm{Kalb}$ & $\left\{\begin{array}{l}3,125 \% \text { frischen Blutes. } \\
3,125 \% \text { des } 24 \text { Stunden alten } \\
\text { Blutes. }\end{array}\right.$ & $\begin{array}{r}6 \\
11\end{array}$ & $\begin{array}{l}1,33467 \\
1,33462\end{array}$ & $\begin{array}{r}+0,00019 \\
+0,00014\end{array}$ \\
\hline Schaaf & $\left\{\begin{array}{l}0,7 \% \text { des frischen Blutes. } \\
3,125 \% \text { des } 48 \text { Stunden alten } \\
\text { Blutes. }\end{array}\right.$ & $\begin{array}{l}20 \\
26\end{array}$ & $\begin{array}{l}1,33474 \\
1,33462\end{array}$ & $\begin{array}{r}+0,00026 \\
+0,00014\end{array}$ \\
\hline Schwein & $\left\{\begin{array}{l}2,1 \% \text { des frischen Blutes. } \\
3,125 \% \text { des } 24 \text { Stunden alten } \\
\text { Blutes. }\end{array}\right.$ & $\begin{array}{l}38 \\
45\end{array}$ & $\begin{array}{l}1,33448 \\
1,33460\end{array}$ & $\begin{array}{l}+0,00000 \\
+0,00012\end{array}$ \\
\hline Ochse & $\left\{\begin{array}{l}6,25 \% \text { frischer Galle. } \\
6,25 \% \text { derselben filtrirten } \\
\text { Galle. }\end{array}\right.$ & $\begin{array}{l}50 \\
55\end{array}$ & $\begin{array}{l}1,33476 \\
1,33536\end{array}$ & $\begin{array}{l}+0,00028 \\
+0,00088\end{array}$ \\
\hline Kuh & $\left\{\begin{array}{l}6,25 \% \text { frischer Galle. } \\
6,2 \% \text { Milch. } \\
6,1 \% \text { Milch. } \\
7,2 \% \text { Milch. }\end{array}\right.$ & $\begin{array}{l}60 \\
65 \\
70 \\
75\end{array}$ & $\begin{array}{l}1,33510 \\
1,33445 \\
1,33500 \\
1,33476\end{array}$ & $\begin{array}{r}+0,00062 \\
+0,00003 \\
+0,00052 \\
+0,00028\end{array}$ \\
\hline
\end{tabular}

Diese Werthe bestätigen von Neuem, dass das Brechungsverhältniss eine weniger empfindliche Eigenschaft, als z. B. die Eigenschwere oder die Blutbänder, bilden. Das Brechungsverhältniss des frischen Schaafblutes näherte sich erst bei weniger als $1 \%$ dem des Wassers nur bis auf drei Einheiten der dritten Decimale.

5. Die Ausflusslehre oder die Newton'sche Emanationstheorie des Lichtes führt zu dem Ausdrucke $\frac{n^{2}-1}{s}$ bei der Darstellung der Wirkung des Snell'schen Brechungsgesetzes ${ }^{1)}$, wenn $n$ das Brechungsverhältniss und $s$ die Eigenschwere oder die mechanische Dichtigkeit bezeichnet. Man nannte daher den Werth $\frac{n^{2}-1}{s}$ das specifische Brechungsvermögen. Biot und Arago nahmen an, dass

1) Siehe z. B. J. C. E. Schmidt, Lehrbuch der analytischen Optik. Göttingen. 1834. S. 318-320, oder die Herleitung in J. C. Poggendorff, Geschichte der Physik. Leipzig 1879. S. 684. 685. 
das Product desselben und des Gewichtes einer tropfbar flüssigen Mischung der Summe der ähnlichen Producte der einzelnen Bestandtheile gleiche. Beschränkt man sich auf die Mengung zweier Flüssigkeiten and nennt $n_{1}$ and $n_{2}$ deren Brechungsverhältnisse, $s_{1}$ und $s_{2}$ deren Eigenschwere, $p_{1}$ and $p_{2}$ die Gewichte derselben, während $n_{3}, s_{3}$ und $p_{3}$ dieselbe Bedeutung für die Mischang haben, so erhält man hiernach :

$$
\frac{n_{3}^{2}-1}{s_{3}} \cdot p_{3}=\frac{n_{1}^{2}-1}{s_{1}} p_{1}+\frac{n_{2}^{2}-1}{s_{2}} \cdot p_{2}
$$

Diese Gleichung lässt sich vereinfachen, wenn man von jeder etwaigen Dichtigkeitsänderung absieht und darauf verzichtet, das specifische Brechungsvermögen in der Formel vorkommen zu lassen. Da das Gewicht $p=v . s$ ist, wenn $v$ den Rauminhalt bezeichnet, so verwandelt sich (1), indem $v_{3}=v_{1}+v_{2}$ ist, in :

$$
n_{3}=\left[1+\frac{\left(n_{1}^{2}-1\right) v_{1}+\left(n_{2}^{2}-1\right) v_{2}}{v_{1}+v_{2}}\right]^{1 / 2}
$$

da $v_{3}=v_{1}+v_{2}$, wenn kein Dichtigkeitswechsel in Folge der Mischung eintritt. Nun ist für unsern Fall $n_{2}=1,33448$, wir erhalten daher:

$$
n_{3}=\left[1+\frac{\left(n_{1}^{2}-1\right) v_{1}+0,78084 v_{2}}{v_{1}+v_{2}}\right]^{1 / 2}
$$

Wird, wie in vielen der hier dargestellten Versuche, $v_{1}=v_{2}$, so geht (3) iiber in:

$$
n_{3}=\left[1+\frac{n_{1}^{2}-0,21916}{2}\right]^{1 / 4}
$$

Will man sich mit einer blossen Annäherung begntïgen, so gibt die binomisehe Entwickelung von (4), wenn man mit dem zweiten Gliede abbricht:

$$
n_{3}=1+\frac{n_{1}^{2}-0,2916}{4}
$$

Landolt ${ }^{1}$ ) wählte den leichter zu berechnenden Werth $\frac{n-1}{s}$ statt $\frac{n^{2}-1}{s}$ in (1) und fand dabei, dass die berechneten und die gefundenen Grössen der Ablenkungsverhältnisse der Misehungen einer Reihe meist ternärer organischen Flüssigkeiten (Weingeist, S. 622.624 .

1) H. Landolt in Poggendorffs Annalen. Bd. 123. Leipzig 1864. 
Aether, Amylalcohol, Aethylaleohol, Essigsänre, Buttersäure, Schwefelkohlenstoff) befriedigend stimmten. E. Forster ${ }^{1}$ ) bestätigte das Gleiche für eine ausgedehnte Anzahl von Lösungen fester Körper (Bromnatrium, Glaubersalz, Natriumnitrat, Borax, Bittersalz, Chlorbaryum, Kalialaun, Kaliumbichromat, Bleinitrat, Silbernitrat).

Nimmt man demgemäss statt (1):

$$
\frac{n_{3}-1}{s_{3}} p_{3}=\frac{n_{1}-1}{s_{1}} p_{1}+\frac{n_{2}-1}{s_{2}} p_{2}
$$

so wird (2) $\mathrm{zu}$ :

$$
n_{3}=1+\frac{\left(n_{1}-1\right) v_{1}+\left(n_{2}-1\right) v_{2}}{v_{1}+v_{2}}
$$

Diese für tropf bare Flïssigkeiten aufgestellte Gleichung (7) würde mit, derjenigen stimmen, welche Biot und Arago und später Dulong ${ }^{2}$ ) für Gasmischungen, deren Bestandtheile keine chemische Verbindung eingehen, annahmen, wenn man das Volumen mit der Masse, also einer dem Gewichte proportionalen Grösse vertauschte.

Wird $v_{1}=v_{2}$, so folgt aus (7)

$$
n_{3}=\frac{n_{1}+n_{2}}{2}
$$

d. h. das Brechnngsverhältniss der Mischung gleicht dem arithmetischen Mittel der Brechungsverhältnisse der beiden gemischten Fliissigkeiten. Schon dieses einfache nnd naturgemässe Ergebniss macht es wahrseheinlich, dass die Gleichung (6) den Erscheinungen mindestens eben so gut als (1) entspricht, ein Schluss, den die Erfahrung, wie wir sogleich sehen werden, bestätigt.

Ist der eine Mischungsbestandtheil destillirtes Wasser von dem Ablenkungsverhältnisse 1,33448, so wird (7) zu:

$$
n_{3}=1+\frac{(n-1) v_{1}+0,33448 v_{2}}{v_{1}+v_{2}}
$$

and für den Fall $v_{1}=v_{2}$ zu:

$$
n_{3}=0,66724+\frac{n_{1}}{2}
$$

1) E. Forster, Untersuchungen über die Beziehungen zwischen dem specifischen Brechungsvermögen und der Concentration von Salzlösungen. Bern 1878. S. $27-38$.

2) Siehe z.B. A. Beer, Einleitung in die höhere Optik. Braunschweig 1853. 8. S. 35. 
Wir wollen nur die je vier ersten hierher gehörenden Bestimmungen des Blutes, der Galle und der Milch zu dem Vergleiche zwischen Rechnung and Erfahrung benutzen, um nicht zu weitlänfig zu werden. Die zwölf Bestimmungen reichen schon zur Rechtfertigung der Hauptschliusse, die wir ziehen werden, hin.

Man findet:

\begin{tabular}{|c|c|c|c|c|c|c|}
\hline \multirow{3}{*}{ 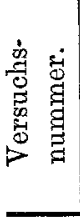 } & \multirow{3}{*}{$\begin{array}{l}\text { Zusammen- } \\
\text { setzang. }\end{array}$} & \multicolumn{5}{|c|}{ Brechungsverhältniss. } \\
\hline & & \multirow{2}{*}{$\begin{array}{c}\text { Ge- } \\
\text { funden. }\end{array}$} & \multicolumn{4}{|c|}{ Berechnet nach dem Ausdrucke von } \\
\hline & & & $\begin{array}{c}\text { Biot und } \\
\text { Arago. }\end{array}$ & Unterschied. & Landolt. & Unterschied. \\
\hline 3 & $\begin{array}{c}25 \% \text { frischen } \\
\text { Kalbsblutes. }\end{array}$ & 1,34396 & 1,34393 & $-0,00004$ & 1,34388 & $-0,00008$ \\
\hline 4 & $12,5 \%$ desselb. & 1,33904 & 1,33925 & $+0,00021$ & 1,33922 & $+0,00018$ \\
\hline 5 & $6,2 \%$ desselben. & 1,33610 & 1,33676 & $+0,00066$ & ],33529 & $-0,00081$ \\
\hline 6 & $3,125 \%$ desselb. & 1,33467 & 1,33529 & $+0,00062$ & 1,33458 & $-0,00009$ \\
\hline 47 & $50 \%$ Galle. & 1,34450 & 1,34462 & $+0,00012$ & 1,34458 & $+0,00008$ \\
\hline 48 & $25 \%$ derselben. & 1,33870 & 1,33950 & $+0,00080$ & 1,33949 & $+0,00079$ \\
\hline 49 & $12,5 \%$ derselb. & 1,33636 & 1,33660 & $+0,00024$ & 1,33660 & $+0,00024$ \\
\hline 50 & $6,25 \%$ derselb. & 1,33476 & 1,33540 & $+3,00064$ & 1,33542 & $+0,00066$ \\
\hline 62 & $49,6 \%$ Milch. & 1,34087 & 1,34152 & $+0,00065$ & 1,34139 & $+0,00052$ \\
\hline 63 & $24,7 \%$ derselb. & 1,33640 & 1,33606 & $+0,00034$ & 1,33606 & $+0,00034$ \\
\hline 64 & $12,4 \%$ derselb. & 1,33471 & 1,33473 & $+0,00002$ & 1,33462 & $+0,00009$ \\
\hline 65 & $\| 6,2 \%$ derselben. & 1,33445 & 1,33459 & $+0,00014$ & 1,33459 & $+0,00014$ \\
\hline
\end{tabular}

Wir sehen hieraus, dass bejde Berechnungsarten nahezu die gleichen Ergebnisse lieferten, was sich auch theoretisch erklärt, wenn man bedenkt, dass die Werthe der hier behandelten Brechungsverhältnisse zwischen 1,33 und 1,36 liegen. Man wird aber die Landolt'sche Ausdrucksweise und bei Gleichheit der Volumina das aus ihr folgende arithmetische Mittel vorziehen, weil diese Rechnung leichter und kürzer ist.

Die Unterschiede zwischen Berechnung and Beobachtung liegen zwischen 0,00002 und 0,00081 nach den Formeln von Biot und Arag 0 und zwischen 0,00008 und 0,00081 nach denen von Landolt. Sie erreichen also nie eine Einheit der dritten Decimale des Brechungsverhältnisses, was der möglichen Genauigkeit der Refractometerbeobachtungen entspricht. Wenn die Rechnung fast immer etwas höhere Werthe als die Erfahrung gibt und hierdurch auf eine beständige Fehlerquelle hinweist, so dürfte dieses denselben Grund haben, wie er oben für die ähnliche Erscheinung, welche die Eigenschwere darbietet, dargestellt worden. 